\title{
Gene expression analysis reveals that formation of the mouse anterior secondary palate involves recruitment of cells from the posterior side
}

\author{
QUN LI and JIXIANG DING* \\ Department of Molecular, Cellular \& Craniofacial Biology and Birth Defects Center, University of Louisville, Kentucky, USA
}

\begin{abstract}
Cleft palate is a common birth defect caused by disruptions in secondary palate development. Anterior-posterior (A-P) regional specification plays a critical role in palate development and fusion. Previous studies have shown that at the molecular level, the anterior palate can be defined by the expression of Shox-2 and the posterior palate by Meox-2 expression in certain mouse strains. Here, we have extended previous studies by performing a more detailed analysis of these genes during mouse palate development. We found that the expression patterns of Shox-2 and Meox-2 are dynamic during palate development. At embryonic day 12.5 (E12.5), Shox-2 expression is localized to the anterior end and its expression domain covers less than $25 \%$ of the length of the palate shelf. The Shox-2 expression domain then gradually expands towards the posterior end and ultimately occupies more than $60 \%$ of the palate shelf by E14.5. The expansion of the Shox-2 domain may involve induction of Shox2 expression in additional cells. Reciprocally, the Meox-2 expression domain at E12.5 covers a large portion of the palate shelf, a region more than $70 \%$ of the entire palate, but then regresses to the posterior $25 \%$ by E14.5. This regression is likely caused by the repression of Meox-2 expression in certain Meox2 expressing cells, rather than the cessation of cell proliferation. Therefore, certain Meox-2 positive "primitive posterior cells" are differentiated/converted into Shox-2 positive "definitive anterior cells" during A-P regional specification.
\end{abstract}

KEY WORDS: Shox-2, Meox-2, secondary palate, regional specification, craniofacial development

During mammalian embryogenesis, the formation of the continuous secondary palate between the oral and nasal cavities involves multiple developmental steps that lead to the fusion of the two bilateral palate shelves along the facial midline (Ferguson 1988; Murray and Schutte, 2004; Nawshad et al., 2004). At the histological level, a developing mouse secondary palate shelf contains a block of neural crest derived mesenchymal cells surrounded by a multi-layered epithelial sheet that originates from the facial ectoderm (Ferguson 1988). In mouse development, the prospective palatal mesenchymal cells begin to be specified in the maxillary processes at embryonic day 11.5 (E11.5) (Murray and Schutte, 2004). The developing palate shelves first grow down vertically along the two sides of the tongue between E12.5 and E13.5. From E14.5, however, the two palatal shelves elevate above the level of the dorsal tongue. The two elevated palatal shelves will continue to grow horizontally and meet each other along the facial midline (Ferguson 1988; Murray and Schutte, 2004; Nawshad et al., 2004). The contact of the two palate shelves at their medial edge epithe- lium (MEE) regions induces fusion of the two palate shelves and the formation of a continuous palate by E15.5 (Shuler et al., 1991; Shuler et al., 1992; Carette and Ferguson, 1992; Griffith and Hay 1992). The proximal-distal growth of the palate shelf is crucial for midline contact. Following contact, the two medial edge epithelial sheets merge to form the MEE seam that will soon undergo degeneration (Ferguson 1988; Shuler et al., 1991; Shuler et al., 1992; Carette and Ferguson 1992; Griffith and Hay 1992; Murray and Schutte, 2004). This process requires the differentiation of palatal medial edge epithelial cells that are distinct from the epithelial cells on oral and nasal sides (Ferguson et al., 1984). Moreover, tissue recombination experiments revealed that the differentiation of medial epithelial cells is determined by the underlying medial edge mesenchymal cells (Ferguson et al., 1984). In addition to proximal-distal growth and medial-lateral differentiation, the palate shelves

Abbreviations used in this paper: A-P, anterior-posterior; MEE, medial edge epithelium; PS, palate shelves.


Louisville, KY 40202, USA. Tel: +1-502-852-2455. Fax: +1-502-852-4702. e-mail: j0ding03@gwise.louisville.edu
} 
also grow and differentiate in the anterior-posterior $(A-P)$ direction. The A-P differentiation at the morphological and cellular levels has long been recognized as the palate shelf displays distinct characterizations along its A-P axis; In particular, the anterior palate is committed to be the bony hard palate, whereas the posterior region will form the soft palate that is composed primarily of smooth muscle (Cui et al., 2005). Only recently, however, has the importance of $A-P$ regional specification in palate fusion been appreciated at the molecular level (Hilliard et al., 2005). The mouse homeobox gene Msx 1 is expressed only in the anterior palatal mesenchymal cells (Zhang et al., 2002; Hilliard et al., 2005) and the loss of MsX1 function in mice results in a complete cleft palate rather than only an anterior cleft, indicating the importance of anterior palate development in palate fusion (Satokata and Maas, 1994; Zhang et al., 2002; Hilliard et al., 2005). Furthermore, the cleft palate caused by $M s \times 1$ inactivation can be rescued by trans-expression of the BMP4 gene (Zhang et al., 2002; Hilliard et al., 2005). Interestingly, in vitro explant culture showed that only the anterior, but not the posterior, palatal mesenchymal cells can proliferate in response to the addition of BMP (Zhang et al., 2002; Hilliard et al., 2005). The expression of Pax9 is restricted to the posterior region during palate development and disruption of this gene in mice results in cleft palate (Peters et al., 1998; Hilliard et al., 2005). All of these experimental results indicate that A-P differentiation and regional specification play critical roles in palate development and fusion. However, our current understanding of the A-P regional specification at the molecular level is limited and several fundamental issues remain to be addressed. For example, the chronology of palatal A-P regional specification has not been examined in detail and it is assumed that the anterior and posterior palates initiate simultaneously and develop synchronously.

In this study, we analyzed three markers, Msx 1, Shox-2and Meox-2, to examine the A-P regional specification during palate development. Mouse Shox-2 gene belongs to a recently identified paired-related homeobox gene family that also found in humans and chicken (Blaschke et al., 1998; Semina et al., 1998; Clement-Jones et al., 2000; Cobb et al., 2006; Tiecke et al., 2006). The founding member of this family, human SHOX gene, is associated with bone related short-stature disorders including Turner syndrome and Leri-Weill syndromes (Clement-Jones et al., 2000). Human SHOX2 gene shares $80 \%$ similarity with human SHOX gene at amino acid level in the homeodomain and C-terminal regions (Blaschke et al., 1998; Semina et al., 1998). In mouse, only one Shox family member, Shox-2, has been identified to date (Yu et al., 2005; Cobb et al., 2006). The amino acid sequences between human and mouse Shox-2 proteins are 99\% identical (Blaschke et al., 1998; Semina et al., 1998). Unlike SHOX, the association of SHOX2 gene with human disease has not been reported. However, studies with mouse embryos indicate that Shox-2 function is also required for bone growth, at least, in limb system development (Cobb et al., 2006). Inactivation of Shox-2 specifically in mouse limb buds leads to severe chondrogenesis defects in long-bone development (Cobb et al., 2006). Further analysis
E12.5

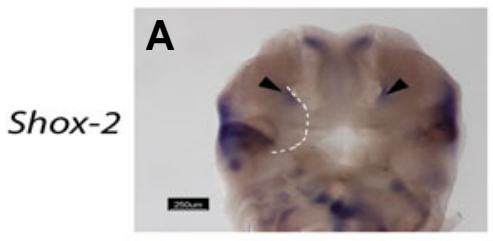

D

Meox-2

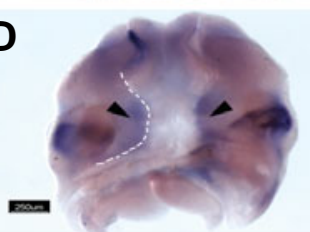

Msx-1

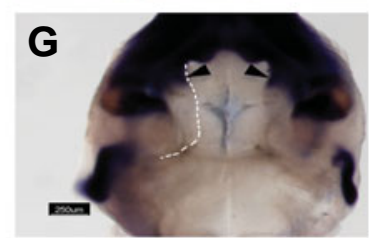

E13.5

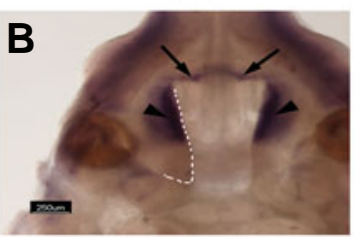

E


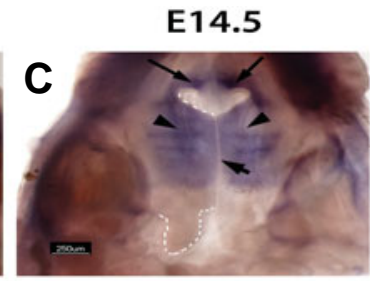

政
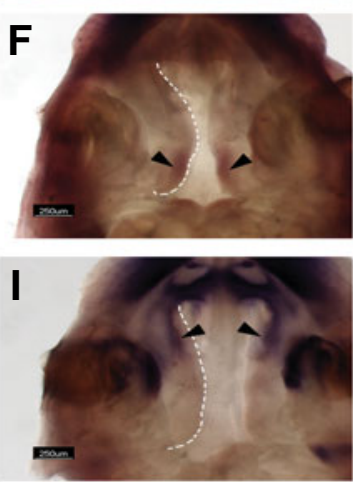

Fig. 1 (Left). Whole mount in situ hybridization showing the expression of Shox-2, Meox-2 and $M s \times 1$ during mouse secondary palate development from E12.5 to E14.5. (A-C) Expression of Shox2 in the secondary palate shelves (arrowheads) and primary palate (arrows) at (A) E12.5, (B) E13.5 and (C) E14.5. The expression is absent from the medial edge epithelium (short arrow) at (C) E14.5. (D-F) Expression of Meox-2 in the secondary palate shelves (arrowheads) at (D) E12.5, (E) E13.5 and (F) E14.5. (G-I) Expression of Msx1 in the secondary palate shelves (arrowheads) at (G) E12.5, (H) E13.5 and (I) E14.5. Scale bars represent 250 um. The white dotted lines indicate the edge of the palate shelf.

Fig. 2 (Right). Comparison of Shox-2 and Msx $\mathbf{1}$ expression domains in palate shelves. (A) Whole-mount views of dissected E12.5 palate shelves showing that the Msx1 expression domain is larger than the Shox-2 expression domain at this stage. (B) Whole-mount views of dissected E13.5 palate shelves showing that the expanded Shox-2 domain covers more area than the Msx1 expression domain. Scale bars represent 250 um. 
indicated that Shox-2functions upstream of Runx2during chondrogenesis (Cobb et al., 2006). In addition to the limb, Shox-2 is also highly expressed in craniofacial region including the secondary palate (Hilliard et al., 2005; Yu et al., 2005). Interestingly, the expression of Shox-2 in the palate is restricted only to the anterior region (Hilliard et al., 2005; Yu et al., 2005). Loss of Shox2function alters FGF signaling and leads to cleft only in the anterior palate (Yu et al., 2005).

Meox-1 and Meox-2define a subfamily of murine homeobox genes with mesoderm and mesenchyme-specific expression during development (Candia et al., 1992). Gene targeting experiments have demonstrated that Meox-2 is required for mouse limb muscle development and that Meox-1 and Meox-2 play an overlapping and essential role during early skeletal morphogenesis (Mankoo et al., 1999; Mankoo et al., 2003). We have previously reported that Meox2 expression in mouse secondary palate is restricted to the posterior end in the MF1 and C57 BL/6 strains (Jin and Ding, 2006).

The specific expression of Shox2 and Msx 1 in the anterior palate and Meox-2 in the posterior palate prompted us to use these markers to examine the A-P regional specification during mouse palate development.

\section{Results and Discussion}

\section{Regional specific expression of Shox-2, Meox-2 and Msx1 in mouse secondary palate development}

Previous studies reported the expression of Meox-2(Jin and Ding, 2006) and Shox2 (Yu et al., 2005) during mouse palate development. In the current study, we analyzed the expression of these two genes by whole mount in situ hybridization in C57BL/6 mouse embryonic heads from E12.5 to E14.5, focusing on the sizes of the expression domains at different stages. As shown in Fig. 1, both Shox2 and Meox-2 display dynamic expression patterns during palate development. At E12.5, the expression of $S$ hox 2 is localized within a small anterior region, an area less than $25 \%$ of the entire palate (Fig. $1 \mathrm{~A}$ ). However, the Shox2expression domain undergoes a dramatic expansion towards the posterior direction at E13.5 and occupies the anterior one-half of the palate (Fig. 1B). This directional expansion of the Shox2expression domain continues into E14.5 and results in the expression of Shox 2 in more than $60 \%$ of the palate (Fig.1C). The expression is predominately in mesenchymal cells (see Fig. 3B). The expression is absent in the MEE region at E14.5 (Fig. 1C). In addition to the secondary palate, Shox 2 expression is also found in the primary palate (Fig.1B and C). In marked contrast to Shox2expression, the expression of Meox-2 at E12.5 covers a large portion (over $70 \%$ ) of the palate shelf with only a small area in the anterior region that is negative for Meox-2 expression (Fig.1D). At E13.5, however, the expression of Meox-2 significantly regresses to the poste- rior end and occupies only $40 \%$ of the palate in this region (Fig. $1 \mathrm{E})$. The regression of the Meox-2 expression domain continues into E14.5 and the size of the Meox-2 expression domain shrinks to about $25 \%$ of the palate, a region corresponding to the future soft palate (Fig. 1F). The expression is strictly mesenchyme-specific (Jin and Ding, 2006).

$M s \times 1$ is another gene reported to display anterior specific expression during palate development (Zhang et al., 2002; Hilliard et al., 2005). In contrast to Shox2expression, our whole mount in situ hybridization data shows that MsX1 expression undergoes modest changes from E12.5 to E14.5 with respect to the A-P axis of the palate (Fig. 1G-I). At E12.5, the expression of $M s \times 1$ covers roughly $25 \%$ of the palate and is restricted to the anterior region (Fig.1G). The expression domain increases to about $30 \%$ of the length of the palate on E13.5 and E14.5 (Fig. $1 \mathrm{H}$ and $\mathrm{I})$. The actual size of the Msx 1 expression domain does increase, but the increase roughly fits the growth of palate. It is worth noting that previous studies have shown that Meox-2and MsX1 are expressed only in the mesenchymal cells (Zhang et al., 2002; Hilliard et al., 2005; Jin and Ding, 2006), whereas the expression of Shox2 is initially (up to E12.5) only in mesenchymal cells and then extends to epithelial cells from E13.5 (Hilliard et al., 2005; Yu et al., 2005).

On E12.5, the area of the Msx 1expression domain is at least equal to the area of the Shox2 expression domain, if not larger (Fig.2 A). However, as a result of the Shox2 expansion, the Shox 2 expression domain is larger than the Msx 1 expression domain on E13.5 (Fig. 2B).

\section{The shifts of Shox2 and Meox-2 expression domains are not due to differential cell proliferation rates between the anterior and posterior palate}

There are at least two possible interpretations for the dynamic expression patterns of Shox2 and Meox-2during palate development: 1) the anterior palate has a significant growth advantage over the posterior palate which leads to the expansion of anterior palate and the relative shrinkage of posterior 


\section{Meox-2}



Fig. 4. Repression of Meox-2 expression and induction of Shox-2 expression during palate development. (A,B) Palate shelves are arranged with the anterior side facing up. (A) The actual size of the Meox2 expression domain at E13.5 is shorter than that at E12.5. (B) The actual size of the non-shox-2 expressing region is reduced during palate development. Scale bars represent (A) $100 \mu \mathrm{m}$ and (B) $250 \mu \mathrm{m}$.

palate; 2) The anterior and posterior palates have a similar growth rate, but a portion of Meox-2 expressing cells are switched to be Shox2 expressing cells during palate development.

To investigate the basis of Shox2 expansion and Meox-2 regression, we carried out a bromodeoxyuridine (BrdU) incorporation assay to examine the cell proliferation status along the $A-P$ axis of the mouse palate in sagittal sections. Since the palate shelf prior to E14.5 is growing vertically, we dissected E13.25 mouse palates and placed them on filter paper in order to obtain sagittal sections with the correct orientation (Fig. 3A). Adjacent sections were cut and processed for the BrdU assay and for in situ hybridizations with Shox2and Meox-2antisense RNA probes. As shown in figure 3, the Shox2and Meox-2expressing regions gave similar intensities of BrdU signals (Fig. 3B-D), suggesting the cell proliferation rates in Shox 2 and Meox-2 expressing regions are similar. To further determine the relative cell proliferation rates in the two regions, we went to determine the density of BrdU positive cells in the two regions by normalizing the BrdU positive cell number to size. It appeared that the densities of BrdU positive cells in the two regions are almost identical. Nevertheless, the

E13.5
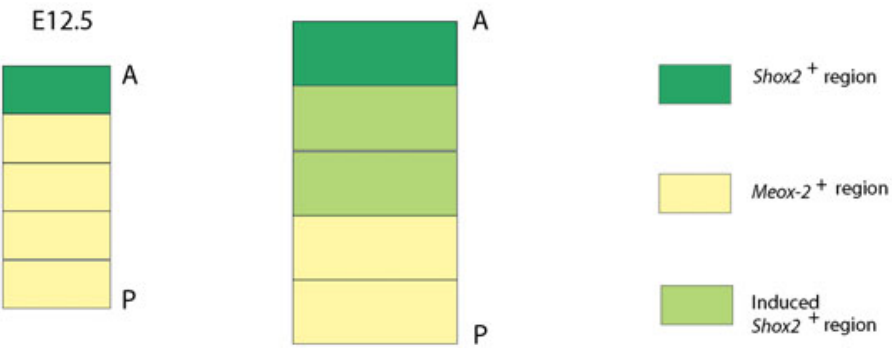

Fig. 5. A schematic representation of the expansion of anterior palate. On E12.5, most of the palatal mesenchymal cells are considered to be posterior cells as defined by the expression of Meox-2, a posterior marker and the absence of Shox-2, an anterior marker. From E12.5 to E13.5, the expansion of the anterior palate occurs, during which a portion of original Meox-2 expressing posterior cells convert to the anterior cells by expressing Shox-2. Abbreviations: $A$, anterior; $P$, posterior. cells in cells the Meox-2 expressing area are highly proliferating. Therefore, the actual size of Meox-2 expression domain should, in principle, increase unless a percentage of Meox-2 expressing cells lose their expression and vice versa, the actual size of nonShox 2 expression domain should also increase unless some of them are differentiated into Shox2 expressing cells.

We next directly compared the Shox2and Meox-2expression domains in palates among different stages. As shown in figure 4, the actual size of the Meox-2expression domain at E13.5 is shorter compared to E12.5 palates (Fig.4A), although the palatal cells are highly proliferative at this stage as shown by the BrdU assay and the E13.5 palate is longer than the E12.5 palate. This result indicates that a certain portion of Meox-2 expressing cells undergo gradual repression of Meox2 expression during palate development. Similarly, the actual size of the non-Shox2 expressing region at E14.5 is shorter than the Shox2 negative regions on E13.5 and E12.5 (Fig. 4B), indicating that some additional cells acquire Shox2expression during palate development.

It has long been recognized by morphology and histology that a well developed mammalian secondary palate consists of an anterior bony palate and posterior muscular soft palate (Ferguson 1988; Cui et al., 2005; Hilliard et al., 2005). Our current study revealed that the anterior and posterior palate regions change dynamically during development at least by molecular measurement. From E12.5 and E13.5, for example, a portion of Meox-2+ / Shox 2 cells are induced or converted to be Shox2t / Meox-2 cells (Fig. 5). We propose that at early stages (prior to E12.5), the majority of palatal mesenchymal cells are more like posterior cells at the molecular level since they are positive for Meox-2, a posterior marker and negative for the anterior markers, Shox2 and Msx1. Here, we refer to those early Meox-2t/Shox 2 cells as "primitive posterior cells". During the expansion of the anterior palate, a portion of original Meox-2 expressing "primitive posterior cells" differentiate to become definitive anterior palatal cells by switching from Meox2 expression to Shox2 expression. Thus, the formation of the anterior palate may involve two steps: 1) establishment of A-P polarity; and 2) extend further anterior differentiation posteriorly.

Previous studies have shown that both Meox-2 and Shox2 expression domains cover the whole palate region from proximal to distal from E12.5 (Yu et al., 2005; Jin and Ding, 2006). The expansion of Shox2and Meox2expression domains in the proximal-distal direction is likely due to cell proliferation.

Since little is known about the down stream target genes for Shox2 and Meox2 at this moment, further studies are required to uncover the molecular mechanism underlying the switch from Meox-2 expressing to Shox 2 expressing. It is possible, however, that the anterior genes such as Shox2 encode transcription repressors that suppress the posterior gene expression.

Although our explanation for the regression of Meox2 expression is repression of Meox-2 expression in certain Meox-2 expressing cells, it is also possible that the regression is caused by posterior migrations or movements of Meox-2 expressing cells. However, the cell migration and movement of palate mesenchymal cells have not yet been demonstrated experimentally. Since no apoptosis has been observed in palate 
mesenchymal cells from E12.5 to E14.5, the regression of the Meox-2 expression domain is not likely due to apoptosis.

\section{Experimental Procedures}

\section{Mice}

As reported in our previous study, the regional specific expression of Meox-2 was observed in C57BL/6 and MF1 mice. The Meox-2 expression does not show regional specificity in certain mouse strains such as Swiss Webster. Therefore, all the mice used in this study are C57BL/6 from Taconic.

\section{Examination of gene expression and the size of expression do- mains}

C57BL/6 mouse embryos were dissected in cold PBS on E12.5, 13.5 and 14.5 (the day when vaginal plugs were observed was designated as day E0.5.). The lower jaw and a piece of brain tissue were cut off and the remaining tissue was processed for non-radioactive whole mount in situ hybridization using digoxygenin-labeled antisense riboprobes as previously described (Jin and Ding, 2001). The results were examined under a Nikon SMZ1500 dissecting microscope and photographed using a Nikon DXM1200F digital camera. To measure the size of expression domain, we imported the image into Adobe Photoshop and used its digital ruler to determine the relative size of the expression domains and its percentage of the entire palate. For section in situ hybridization, the palatal shelves were dissected and sagittal sections were prepared as described below. $12 \mu \mathrm{m}$ cryosections were cut and air-dried overnight before the in situ hybridization was carried out (Jin and Ding, 2006). The results of section in situhybridization were scored under a Nikon E600 microscope.

\section{Preparation of palatal sagittal sections}

To obtain palatal sagittal sections with correct orientation prior to $E 14.5$, palatal shelves at E13.25 were dissected out and briefly placed on filter paper before fixation (Fig. 3A). The fixed palate shelves were dehydrated in $30 \%$ sucrose in PBS overnight before O.C.T embedding. The filter papers were removed during the embedding.

\section{Cell proliferation assay}

Cell proliferation was determined by a bromodeoxyuridine (BrdU) incorporation assay. Briefly, pregnant female mice were injected with $\mathrm{BrdU}$ (Sigma) at $200 \mathrm{mg} / \mathrm{kg}$ and euthanized 2 hours later and embryos were collected. The palatal shelves were dissected and prepared for sagittal cryo-section as described above. Incorporation of BrdU was determined by immunocytochemistry using an anti-BrdU antibody from BD Bioscience (Cat \# 347580). To determine the density of BrdU positive cells, the numbers of BrdU positive cells in Shox-2 and Meox2 expressing areas were counted separately and the size of each region was measured in Adobe Photoshop.

\section{Acknowledgements}

We thank Dr. William Young and Dr. Dennis Warner for comments on the manuscript and Dr. Jiu-Zhen Jin for her assistance. J.D. was a recipient of American Heart Association Scientist Development Award (0130461T) and Leukemia \& Lymphoma Society Special Fellowship (3402-02). This work is supported by COBRE program of the National Center for Research Resource (P20RR017702 to the University of Louisville Birth Defects Center) and National Institutes of Health (DE015565 and DE016845 to J.D.).

\section{References}

BLASCHKE, R.J., MONAGHAN, A.P., SCHILLER, S., SCHECHINGER, B., RAO, E., PADILLA-NASH, H., RIED, T. and RAPPOLD, G.A. (1998) SHOT, a
SHOX-related homeobox gene, is implicated in craniofacial, brain, heart and limb development. Proc.Nat..Acad.Sci.U.S.A 95: 2406-2411.

CANDIA, A.F., HU, J., CROSBY, J., LALLEY, P.A., NODEN, D., NADEAU, J.H. and WRIGHT, C.V. (1992) Mox-1 and Mox-2 define a novel homeobox gene subfamily and are differentially expressed during early mesodermal patterning in mouse embryos. Development 116: 1123-1136.

CARETTE, M.J. and FERGUSON, M.W. (1992) The fate of medial edge epithelial cells during palatal fusion in vitro: an analysis by Dil labelling and confocal microscopy. Development 114: 379-388.

CLEMENT-JONES, M., SCHILLER, S., RAO, E., BLASCHKE, R.J., ZUNIGA, A., ZELLER,R., ROBSON, S.C., BINDER, G., GLASS, I., STRACHAN, T., LINDSAY, S. and RAPPOLD, G.A. (2000) The short stature homeobox gene SHOX is involved in skeletal abnormalities in Turner syndrome. Hum.Mol.Genet. 9: 695-702.

COBB, J., DIERICH, A., HUSS-GARCIA, Y. \& DUBOULE, D. (2006) A mouse model for human short-stature syndromes identifies Shox2 as an upstream regulator of Runx2 during long-bone development. Proc.Natl.Acad.Sci.U.S.A 103: 4511-4515.

CUI, X.M., SHIOMI, N., CHEN, J., SAITO, T., YAMAMOTO, T., ITO, Y., BRINGAS, P., CHAI,Y. and SHULER, C.F. (2005) Overexpression of Smad2 in Tgfbeta3-null mutant mice rescues cleft palate. Dev.Biol. 278: 193-202.

FERGUSON, M.W. (1988) Palate development. Development 103 Suppl: 41-60.

FERGUSON, M.W., HONIG, L.S. and SLAVKIN, H.C. (1984) Differentiation of cultured palatal shelves from alligator, chick and mouse embryos. Anat.Rec., 209: 231-249.

GRIFFITH, C.M. and HAY, E.D. (1992) Epithelial-mesenchymal transformation during palatal fusion: carboxyfluorescein traces cells at light and electron microscopic levels. Development 116: 1087-1099.

HILLIARD, S.A., YU, L., GU, S., ZHANG, Z. and CHEN, Y.P. (2005) Regional regulation of palatal growth and patterning along the anterior-posterior axis in mice. J.Anat. 207: 655-667.

JIN, J.Z. and DING, J. (2006) Analysis of Meox-2 mutant mice reveals a novel postfusion-based cleft palate. Dev.Dyn. 235: 539-546.

MANKOO, B.S., COLLINS, N.S., ASHBY, P., GRIGORIEVA, E., PEVNY, L.H., CANDIA, A., WRIGHT, C.V., RIGBY, P.W. and PACHNIS, V. (1999) Mox2 is a component of the genetic hierarchy controlling limb muscle development. Nature 400: 69-73.

MANKOO, B.S., SKUNTZ, S., HARRIGAN, I., GRIGORIEVA, E., CANDIA, A., WRIGHT,C.V., ARNHEITER, H. and PACHNIS,V. (2003) The concerted action of Meox homeobox genes is required upstream of genetic pathways essential for the formation, patterning and differentiation of somites. Deve/opment 130: 4655-4664.

MURRAY, J.C. and SCHUTTE, B.C. (2004) Cleft palate: players, pathways and pursuits. J.Clin. Invest 113: 1676-1678.

NAWSHAD, A., LAGAMBA, D. and HAY, E.D. (2004) Transforming growth factor beta (TGFbeta) signalling in palatal growth, apoptosis and epithelial mesenchymal transformation (EMT). Arch. Oral Biol. 49: 675-689.

PETERS, H., NEUBUSER, A., KRATOCHWIL, K. and BALLING, R. (1998) Pax9deficient mice lack pharyngeal pouch derivatives and teeth and exhibit craniofacial and limb abnormalities. Genes Dev. 12: 2735-2747.

SATOKATA, I. and MAAS, R. (1994) Msx1 deficient mice exhibit cleft palate and abnormalities of craniofacial and tooth development. Nat.Genet. 6: 348-356.

SEMINA, E.V., REITER, R.S. and MURRAY, J.C. (1998) A new human homeobox gene OGI2X is a member of the most conserved homeobox gene family and is expressed during heart development in mouse. Hum.Mol.Genet. 7: 415422.

SHULER, C.F., GUO, Y., MAJUMDER, A. and LUO, R.Y. (1991) Molecular and morphologic changes during the epithelial-mesenchymal transformation of palatal shelf medial edge epithelium in vitro. Int.J.Dev.Biol. 35: 463-472.

SHULER, C.F., HALPERN, D.E., GUO, Y. and SANK, A.C. (1992) Medial edge epithelium fate traced by cell lineage analysis during epithelial-mesenchymal transformation in vivo. Dev. Biol. 154: 318-330.

TIECKE, E., BANGS, F., BLASCHKE, R., FARRELL, E.R., RAPPOLD, G. and TICKLE,C. (2006) Expression of the short stature homeobox gene Shox is restricted by proximal and distal signals in chick limb buds and affects the 
length of skeletal elements. Dev.Biol. (on line).

YU, L., GU, S., ALAPPAT, S., SONG, Y., YAN, M., ZHANG, X., ZHANG, G., JIANG, Y., ZHANG, Z., ZHANG, Y. and CHEN, Y. (2005) Shox2-deficient mice exhibit a rare type of incomplete clefting of the secondary palate. Development 132: 4397-4406.

ZHANG, Z., SONG, Y., ZHAO, X., ZHANG, X., FERMIN, C. and CHEN, Y. (2002) Rescue of cleft palate in Msx1-deficient mice by transgenic Bmp4 reveals a network of BMP and Shh signaling in the regulation of mammalian palatogenesis. Development 129: 4135-4146.

Received: 10th August 2006 Reviewed by Referees: 7th September 2006 Modified by Authors and Accepted for Publication: 13th October 2006 Published Online: 2nd February 2007 